\title{
Effect of surfactant on pharyngeal mechanics in sleeping humans: implications for sleep apnoea
}

\author{
M.J. Morrell*, Y. Arabi", B.R. Zahn ${ }^{\ddagger}$, K.C. Meyer", J.B. Skatrud", M. Safwan Badr"
}

Effect of surfactant on pharyngeal mechanics in sleeping humans: implications for sleep apnoea. M.J. Morrell, Y. Arabi, B.R. Zahn, K.C. Meyer, J.B. Skatrud, M. Safwan Badr. (C) ERS Journals Ltd 2002.

ABSTRACT: Instillation of surfactant into the pharyngeal lumen reduces the pressure required to reopen an occluded airway, and decreases the apnoea/hypopnoea index (AHI). The authors hypothesised that surfactant also reduces the sleep-related increase in pharyngeal resistance. To test this hypothesis two single blind, crossover, placebocontrolled studies were performed.

In protocol A seven male, asymptomatic snoring subjects were studied during sleep. Inspiratory pharyngeal resistance was calculated from plots of airflow versus supraglottic pressure (seven breaths) before and after surfactant or saline instillation. In protocol B, in a different group of seven male subjects with sleep apnoea (AHI 15.2 (12) events $\cdot h^{-1}$ ) the effect of surfactant or saline on sleep disordered breathing was measured, for $1 \mathrm{~h}$ immediately before and after surfactant or saline instillation.

Surfactant decreased pharyngeal resistance calculated at peak pressure (group mean (SD): pre versus post $83.7(76.4)$ versus $\left.49.4(71.1) \mathrm{cmH}_{2} \mathrm{O} \cdot \mathrm{L}^{-1} \cdot \mathrm{s}^{-1}\right)$ and significantly reduced the respiratory disturbance index (RDI pre versus post 79.7 (58.7) versus 59.6 (56.9) events $\cdot h^{-1}$ ). Saline did not decrease resistance (pre versus post 58.6 (31.1) versus $72.5(73.4) \mathrm{cmH}_{2} \mathrm{O} \cdot \mathrm{L}^{-1} \cdot \mathrm{s}^{-1}$ ) or RDI (pre versus post $75.3(42.4)$ versus 79.9 (46.1) events $\left.\cdot h^{-1}\right)$.

Surfactant reduced the collapsibility of the pharynx and led to a modest reduction in respiratory disturbance index. The authors speculate that surfactant may delay occlusion by reducing the liquid "bridging" within the folded pharyngeal lining. Eur Respir J 2002; 20: 451-457.
*National Heart and Lung Institute, Imperial College School of Medicine, Sleep and Ventilation Unit, Royal Brompton Hospital, London, UK. \#Middleton Veterans Administration (VA) Hospital and the University of Wisconsin, Madison, WI and "Detroit VA Medical Centre, Wayne State University, Detroit, MI, USA.

Correspondence: M.J. Morrell, Sleep and Ventilation Unit, Royal Brompton Hospital, London, SW3 6NP, UK. Fax: 442073518911

E-mail: m.morrell@ic.ac.uk

Keywords: Apnoea, breathing, human, sleep, surfactant

Received: August 172001

Accepted after revision: March 152002

This study was supported by the Veterans Adminisration Medical Research Services and National Heart, Lung and Blood Institute. M.J. Morrell is supported by a Wellcome Trust Career Development Fellowship
The influence of mucosal factors on pharyngeal lumen calibre in patients with obstructive sleep apnoea is unclear. It has been known for some time that the intraluminal pressure required to reopen an occluded pharynx is higher than that required to collapse the airway [1-4]. The hysteresis in the closing and reopening pressures suggests that surface tension may be important in maintaining airway occlusion during an apnoea. This idea is supported by the finding that the application of surfactant (which reduces surface tension forces) decreases the pressure required to reopen the pharynx [5] and reduces the apnoea/hypopnoea index (AHI) in patients with sleep apnoea [6]. Surface-active agents may also influence upper airway resistance. Indirect evidence for this suggestion is that instillation of surfactant reduces snoring in humans [6] and in anaesthetised dogs instillation of surface-active fluid (Sonarex) decreases upper airway resistance [7, 8] and reduces snoring [9].

In the present study, the effect of instillation of surfactant during sleep on upper airway mechanics and sleep disordered breathing has been investigated. The authors hypothesised that surfactant would reduce inspiratory pharyngeal resistance by reducing airway narrowing at peak negative pressure, and that this would reduce airflow limitation. The authors also reasoned that this effect, together with a reduction in the pharyngeal re-opening pressure, would stabilise the upper airway and reduce the severity of sleep apnoea. To test this hypotheses two protocols were performed. In protocol A the effect of surfactant on pharyngeal mechanics in snoring subjects with airflow limitation was investigated. In protocol B the amount of sleep disordered breathing during nonrapid eye movement (NREM) sleep, following instillation of surfactant in subjects with sleep apnoea was determined. Natural bovine surfactant was used (Survanta; Ross Laboratories, Columbus, OH, USA), which contained added proteins, including apoprotein $\mathrm{B}$ that acts to anchor the surface tension-lowering phospholipid components to the mucosal surface.

\section{Methods}

\section{Subjects}

Nineteen male asymptomatic snorers were recruited from a database of volunteers to take part in protocol 
A. Nine male patients with sleep apnoea were recruited at random from patients attending the clinical sleep laboratory at the University of Wisconsin Hospital to take part in protocol B.

Before taking part in the study, all subjects were screened to confirm the presence or absence of sleep apnoea and/or snoring. The screening polysomnography consisted of the following measurements: sleep stage, an index of airflow (oral/nasal thermister), ribcage and abdominal effort, snoring (tracheal microphone), oxygen saturation (finger pulse oximeter), and leg electromyogram (EMG). The data from this study was analysed using the standard criteria of the authors' clinical sleep laboratory. Apnoea: cessation of airflow for $>10$ s. Hypopnea: $30 \%$ drop in airflow, plus a drop in oxygen saturation $>4 \%$. Subjects were classified as asymptomatic snorers if they had flow limitation with no excessive daytime sleepiness and an $\mathrm{AHI}<2$ events $\cdot \mathrm{h}^{-1}$. Sleep apnoea syndrome was defined as AHI $>10$ events $\cdot h^{-1}$. All subjects were healthy with no evidence of heart failure or pulmonary disease. The Human Subject Committees of the authors' institutions approved the protocol.

Protocol A: to investigate the effect of surfactant on pharyngeal resistance

Measurements. Sleep was measured using two electroencephalograms (C3-A2, C4-A1), two electrooculograms (left and right eye) and a chin EMG. Electrodes were attached to standard amplifiers (model 7-D; Grass Ltd, Warwick, RI, USA). Surfactant or saline was instilled into the pharynx via a catheter (outer diameter $3 \mathrm{~mm}$ ) which was passed through the nose with the tip visualised at the level of the soft palate. A second transducer-tipped catheter (MPC-500; Millar Instruments, Houston, TX, USA) was used to measure supraglottic pressure. This catheter was passed though the nose, and its tip was positioned $2-3 \mathrm{~cm}$ below the base of the tongue. The position of the catheter was checked visually through the month with the aid of a laryngoscope. Inspiratory and expiratory airflow was measured using a heated pneumotachometer (model 370A; Hans Rudolph, Kansas City, MO, USA) attached to the patient via a nasal mask (medium size, GoldSeal; Resperonics, Kennewick, WA, USA). The dead space of the mask on the patient was $\sim 90 \mathrm{~mL}$ plus pneumotachometer $\sim 14 \mathrm{~mL}$. The relative phase of the pressure and airflow signals was tested prior to the study; there was no appreciable phase lag up to $10 \mathrm{~Hz}$. Oxygen saturation was measured by ear pulse oximetry (model 3740; Ohmeda, Louisville, CO, USA). The respiratory data were acquired at a sampling frequency $20-40 \mathrm{~Hz}$ on two systems, either Biobench (National Instruments, Austin, TX, USA), or MacLab/8e (ADInstruments, Sydney, Australia).

Protocol. Prior to the study, subjects were instructed to restrict their sleep (maximum sleep time 4-6 h). They reported to the sleep laboratory between 19:00 and 20:00 and gave written informed consent. Sleep staging electrodes were then attached and they were asked to lie on the bed. The two catheters were passed into the nose and positioned as explained above. The nasal mask was fitted and the pneumotachometer connected. The mouth was taped to ensure nasal breathing and the pulse oximeter was placed on the ear. Subjects were then allowed to go to sleep, they were observed all night to ensure that they slept only in the supine position.

Saline or surfactant instillation was randomised. Once stage II NREM sleep (or deeper) had been established for $>5 \mathrm{~min}, 1 \mathrm{~mL}$ of surfactant, diluted in $3 \mathrm{~mL}$ of $0.9 \%$ saline was instilled via the pharyngeal catheter over $1 \mathrm{~min}$; $1 \mathrm{~mL}$ of air was used to empty the catheter dead space. Alternatively, $4 \mathrm{~mL}$ of $0.9 \%$ saline were instilled followed by a $1 \mathrm{~mL}$ air flush. The $5 \mathrm{~min}$ immediately following instillation of either surfactant or saline were disregarded, due to arousal and swallowing.

Analysis. Sleep stage and arousals were analysed using standard criteria $[10,11]$, by a researcher blinded to the respiratory data and the interventions. The presurfactant or saline analysis period was taken as the seven breaths immediately prior the instillation. The postsurfactant or saline period was taken as the first period of stable sleep (with no arousals) that matched the pre-instillation sleep state.

To determine the effect of surfactant on upper airway mechanics, airflow was plotted against supraglottic pressure for seven consecutive breaths preand postinstillation of surfactant (or saline). The seven pre-instillation plots were averaged to produce a mean flow/pressure loop for each subject; this was repeated for the postinstillation data. Mean loops were generated for breaths of different duration by normalising the supraglottic pressure and flow. This was done by weighting each data point as a percentage of inspiratory (or expiratory) time e.g. if 50 data points occurred during inspiration each would represent $2 \%$ of the breath. The breath with the shortest inspiratory time was then selected as a template and the percentages for each of the seven breaths in the loop were matched; the extraneous points (when percentages did not match) were removed. A composite loop was created by averaging the value at each percentage point for all seven breaths.

For each individual, upper airway resistance was calculated at peak pressure, peak flow and fixed flow $\left(0.09 \mathrm{~L} \cdot \mathrm{s}^{-1}\right)$ from the flow/pressure loops by dividing the supraglottic pressure by the airflow. Upper airway resistance at a fixed flow $0.09 \mathrm{~L} \cdot \mathrm{s}^{-1}$ was taken as a numerical representation of the linear part of the flow/ pressure plot. The measurement of resistance at peak flow was taken as the highest resistance attainable in a noncollapsible tube with turbulent flow, and at peak pressure was taken as the smallest upper airway dimensions within a breath during airflow limitation. The mean resistances (fixed flow, peak flow and peak pressure) in each subject pre versus postinstillation of surfactant and saline were compared using paired t-tests with significance noted if $\mathrm{p}<0.05$. 
Protocol B: to investigate the effect of surfactant on sleep disordered breathing

Measurements and protocol. The measurements carried out during protocol B were identical to those in protocol A, with the exception of supraglottic pressure which was not measured.

Analysis. For each sleep apnoea patient the respiratory disturbance index (RDI) was calculated for $1 \mathrm{~h}$ prior to the instillation of surfactant and saline, and for the $1 \mathrm{~h}$ following the instillation. To ensure that the broad spectrum of sleep disordered breathing that occurred in this study was accurately measured, an algorithm modified from TAHA et al. [12] was used for analysis. The detection of an event began with an oxygen desaturation $\geqslant 2 \%$ with a rate of descent $>0.1 \% \cdot \mathrm{s}^{-1}$ (but $<4 \% \cdot \mathrm{s}^{-1}$ ). An apnoea was defined as no inspiration for $>5 \mathrm{~s}$, coincident with the desaturation event. A hypopnoea was defined as a minimum of three breaths showing a $>20 \%$ reduction in tidal volume from the immediately preceding breath, followed by a return to $\geqslant 90 \%$ of that "baseline" breath. Using these criteria desaturation events that were not associated with a hypopnoea, were scored separately from apnoeas and hypopnoeas. The total number of respiratory events (apnoeas, hypopnoeas and desaturations) was normalised to the total sleep time to give the RDI (which according to the definitions used was appreciably longer than the conventional AHI). For each individual (one trial per subject) the sleep and breathing patterns were compared pre and postsurfactant and saline using paired t-tests with significance noted if $\mathrm{p}<0.05$.

\section{Results}

\section{Protocol A}

The effect of surfactant on upper airway mechanics during sleep was studied in 19 subjects. Sufficient data were obtained in seven, five of whom were studied on a single night; the other two subjects were studied on two nights (i.e. saline data collected on one night and surfactant data on a separate night); this was necessary because on the first night subjects woke after instillation of the second fluid. Of the twelve subjects who did not complete protocol A, seven were excluded because they were found to have AHI (despite having none during the screening study), two woke up during instillation of fluids, two did not have inspiratory flow limitation on the study night, and one subject moved out of the area before they could be restudied. There was no difference in severity of disease, age or BMI between those subjects who did and did not complete the protocol.

\section{Protocol B}

The effect of surfactant on the RDI was studied in nonsleep apnoeic patients. Sufficient data were obtained in seven, all of whom were studied on two nights with pre and postsaline data collected on a different night to pre and postsurfactant data. Of the two patients who did not complete the protocol, one was unable to tolerate the nasal catheter used to instill the surfactant, and the other was unable to tolerate the nasal mask.

\section{Screening study results}

For the seven snorers studied in protocol A, the group mean (SD) AHI was $0.93(0.45)$ events $\cdot h^{-1}$. For the seven sleep apnoea syndrome patients in protocol $\mathrm{B}$ the AHI was $15.6(12.0)$ events $\cdot \mathrm{h}^{-1}$. Between the two groups (protocols A and B) there were no significant differences in age (snorers 32 (9) yrs, sleep apnoeics 41 (17) yrs; $\mathrm{p}=0.32$ ) or body mass index (snorers 30 (4) $\mathrm{cm} \cdot \mathrm{kg}^{-2}$, sleep apnoeics $\left.29(4) \mathrm{cm} \cdot \mathrm{k}^{-2} ; \mathrm{p}=0.55\right)$.

\section{Protocol A: effect of surfactant and saline on pharyngeal mechanics}

An example of the reduction in airflow limitation during surfactant instillation is shown for one individual (fig. 1). During the presurfactant breaths


Fig. 1.-A composite plot of airflow versus supraglottic pressure in one asymptomatic snorer is shown: a) mean of seven breaths pre (-) and post (- - ) surfactant and b) mean of seven breaths pre $(-)$ and post (- - ) saline instillation. 
Table 1.-Effects of surfactant and saline on pharyngeal resistance

\begin{tabular}{|c|c|c|c|c|c|c|}
\hline & \multicolumn{3}{|c|}{ Surfactant } & \multicolumn{3}{|c|}{ Saline } \\
\hline & Pre & Post & $\mathrm{p}$-value & Pre & Post & p-value \\
\hline $\begin{array}{l}\text { Resistance at peak } \\
\quad \text { pressure } \mathrm{cmH}_{2} \mathrm{O} \cdot \mathrm{L}^{-1} \cdot \mathrm{s}^{-1}\end{array}$ & 83.7 (76.4) & $49.4(71.1)^{*}$ & 0.004 & $58.6(31.1)$ & $72.5(73.4)$ & NS \\
\hline $\begin{array}{l}\text { Resistance at peak } \\
\quad \text { flow } \mathrm{cmH}_{2} \mathrm{O} \cdot \mathrm{L}^{-1} \cdot \mathrm{s}^{-1}\end{array}$ & $23.2(18.7)$ & $12.7(6.0)$ & NS & $15.4(6.3)$ & $17.3(7.8)$ & NS \\
\hline $\begin{array}{l}\text { Resistance at } 0.9 \mathrm{~L}^{-1} \cdot \mathrm{s}^{-1} \\
\quad \text { fixed flow } \mathrm{cmH} \mathrm{H}_{2} \mathrm{O} \cdot \mathrm{L}^{-1} \cdot \mathrm{s}^{-1}\end{array}$ & $14.7(15.8)$ & $3.5(3.2)$ & NS & $4.3(6.1)$ & $5.7(5.8)$ & NS \\
\hline
\end{tabular}

Data are presented as group mean (SD) $(n=7)$; NS: nonsignificant $p>0.05$. *: $p<0.05$.

and the pre and postsaline breaths, peak flow occurred in early inspiration, the subsequent period of airflow limitation was associated with upper airway narrowing that resulted in no increase in airflow when intraluminal pressure was more negative. It was also observed that the airflow oscillations associated with the snoring that occurred in presurfactant and saline conditions were not seen in the postsurfactant period.

In all subjects the postinstillation data were collected 9-11 min after the instillation of surfactant or saline. Surfactant instillation was associated with a significant reduction in resistance measured peak pressure ( $\mathrm{p}=0.004$, power 0.47 ), but not at peak flow $(p=0.13)$ or at fixed flow on the linear portion of the airflow versus pressure plot $(\mathrm{p}=0.11)$ (table 1). Saline instillation produced no significant change in resistance measured at peak pressure, peak flow or fixed flow ( $p=0.49, p=0.32, p=0.72$, respectively). The surfactant-related reduction in resistance at peak pressure was associated with a significant reduction in peak inspiratory pressure $(\mathrm{p}=0.004)$ and an increase in airflow at peak pressure $(\mathrm{p}=0.003)$ (fig. 2$)$.

\section{Protocol B: effect of surfactant and saline on sleep} and breathing patterns

The effect of surfactant and saline on the sleep and breathing patterns is shown in table 2. Neither surfactant nor saline had any significant effect on the sleep quality. Surfactant administration was associated with a small but statistically significant reduction in RDI during NREM sleep ( $\mathrm{p}=0.04$, power=0.51). However, the duration of apnoeas or hypopnoeas and the number of arousals were not influenced by the instillation of surfactant (table 2). Saline administration was not associated with a statistically significant change in any of the parameters measured

\section{Discussion}

The present study was carried out to determine the effect of surfactant on upper airway mechanics and the severity of sleep disordered breathing. The main finding of this study was that instillation of a single dose of surfactant reduced pharyngeal resistance and decreased peak pressure generated in a relatively small sample of subjects with airflow limitation. In addition,
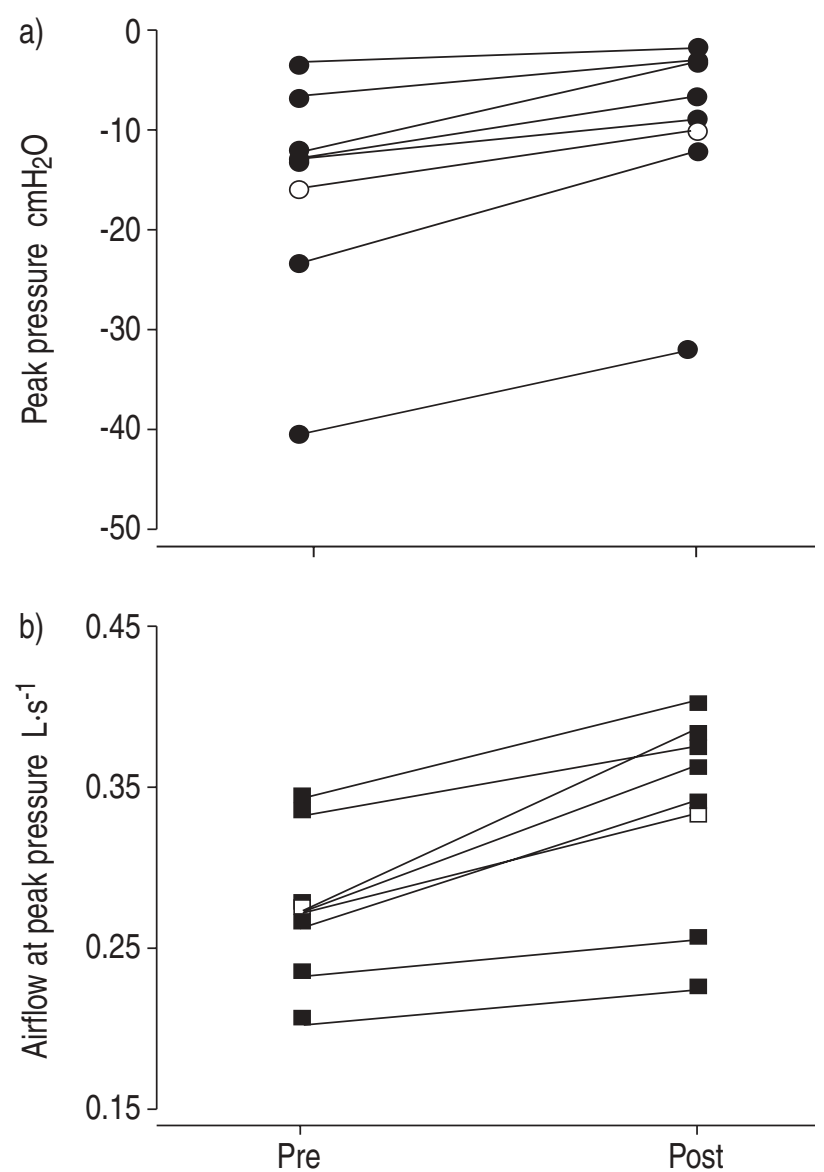

Fig. 2. - Individual mean values (filled symbols) and group means (open symbols) of a) peak pressure and b) airflow at peak pressure pre and post surfactant instillation. Note that surfactant was associated with significantly less negative pressure and an increase in airflow.

instillation of surfactant was associated with a small reduction in sleep disordered breathing in a small sample of sleep apnoeic patients. Taken together these findings suggest that surfactant acts to stabilise the pharyngeal airway, and that instillation of substances that are likely to reduce mucosal surface tension might be a useful adjunctive therapy in people with mild sleep-disordered breathing.

Previous studies in awake humans have shown that when surfactant is applied to the upper airway mucosa a more negative pressure is required to close the 
Table 2. - Effects of surfactant and saline on sleep state and respiratory disturbance index (RDI)

\begin{tabular}{|c|c|c|c|c|}
\hline & \multicolumn{2}{|c|}{ Surfactant } & \multicolumn{2}{|c|}{ Saline } \\
\hline & Pre & Post & Pre & Post \\
\hline $\begin{array}{l}\text { Wakefulness time during } \\
\text { analysis period min }\end{array}$ & $15.8(11.3)$ & $10.4(6.5)$ & $10.6(4.8)$ & $7.0(7.5)$ \\
\hline $\begin{array}{l}\text { Total sleep time during } \\
\text { analysis period min }\end{array}$ & $43.4(11.6)$ & $48.9(7.2)$ & $47.0(5.0)$ & $53.0(7.5)$ \\
\hline NREM Stage I min & $9.9(7.5)$ & $14.3(10.4)$ & $18.1(17.9)$ & $10.9(12.4)$ \\
\hline NREM Stage II min & $28.1(13.6)$ & $31.4(12.1)$ & $22.7(17.0)$ & $32.3(19.1)$ \\
\hline NREM Stage III/IV min & 5.5 (11.6) & $3.1(7.7)$ & $6.1(9.9)$ & $9.8(15.2)$ \\
\hline Arousals total no. & $54.0(23.9)$ & $61.3(34.3)$ & $40.7(3.4)$ & $62.3(36.1)$ \\
\hline Hypopnoeas events $\cdot h^{-1}$ & $36.0(32.5)$ & $24.8(24.0)$ & $37.4(15.9)$ & $48.1(34.7)$ \\
\hline Apnoeas events $\cdot h^{-1}$ & $40.7(58.9)$ & $33.0(44.3)$ & $33.2(42.8)$ & $28.9(35.3)$ \\
\hline Apnoea duration s & $9.9(2.5)$ & $10.4(2.3)$ & $9.1(3.2)$ & $10.7(3.7)$ \\
\hline Hypopnoea duration s & $19.8(2.8)$ & $22.2(4.1)$ & $20.6(3.0)$ & $20.5(4.2)$ \\
\hline Desaturations events $\cdot \mathrm{h}^{-1}$ & $1.9(3.2)$ & $1.8(3.6)$ & $4.7(4.4)$ & $2.7(3.3)$ \\
\hline RDI events $\cdot h^{-1}$ & $79.7(58.7)$ & $59.6(56.9)^{*}$ & $75.3(42.4)$ & $79.9(46.1)$ \\
\hline
\end{tabular}

Data are presented as mean (SD). NREM: nonrapid eye movement sleep. ${ }^{*}$ : $<<0.05$ pre versus post surfactant.

airway [5]. The current authors' findings are the first to measure the effects of surfactant on pharyngeal mechanics in humans during sleep. The reduction in resistance at peak inspiratory pressure was seen in all subjects and is consistent with the speculation that the upper airway is less collapsible and more resistant to closure in the presence of surfactant; although it should be noted that the collapsibility of the upper airway was not directly tested in the present study and that the sample size was small.

The findings that the severity of obstructive sleep apnoea improved following the instillation of surfactant in this study is consistent with another recent study that used a "long-acting tissue lubricant" to produce an improvement in AHI in ten patients with sleep apnoea [6]. It is of note that in both the present study and the study by JoKIC et al. [6] sleep apnoea was not cured in any subject. However, in the study by JOKIC et al. [6] the overall arousal frequency fell while in the present study the number of arousals remained high following the instillation of surfactant and saline. The current authors speculate that this may have occurred due to an increased frequency of "spontaneous" arousals (i.e. not associated with apnoeas and hypopnoeas) caused by the sensation of fluid in the pharynx. The increased number of nonrespiratory arousals in the JoKIC et al. [6] study may support this idea.

\section{Critique of methods}

An important limitation of the present study is that the number of subjects in whom a complete data set could be collected was relatively small, this resulted in the relatively low power of the study. However, the authors think that the observations in this study are important because they showed that during sleep, surfactant produced a significant reduction in resistance at peak flow in all the subjects studied: such observations provide insight into the mechanisms associated with the surfactant-induced reduction in the severity of apnoea.
In the present study upper airway resistance was measured as an index of airway patency. A limitation of this approach is that once airflow limitation is reached the relationship between the supraglottic pressure and airflow is no longer linear and inferences regarding resistance and airway patency are problematic. Nevertheless, inspection of the airflow/pressure loops provide important information regarding upper airway mechanics. Resistance calculated from the linear portion of the loop is an accurate refection of pharyngeal cross sectional area. The resistance calculated at peak flow provides an index of the point at which airflow limitation begins to occur, and the resistance at peak pressure may be related to the smallest upper airway dimensions that occur within a breath during airflow limitation. The authors believe that combining these measurements provides a way of characterising the changes in pharyngeal mechanics associated with the instillation of surfactant, and that changes seen are likely to reflect changes in airway patency. To characterise the effects of surfactant on upper airway patency fully would require imaging studies during sleep.

Measurements of resistance were made during stages II, and deep nonREM sleep (pre and post conditions were always matched). It was necessary to include both stages of nonREM sleep in order to obtain sufficient data across pre and postsurfactant and saline conditions. During stage III/IV sleep the airway is more stable and sleep apnoea is less common, therefore the inclusion of stage III/IV sleep periods in the analysis may have led to an underestimation of the effects of surfactant.

Changes in surface tension following instillation of surfactant were not measured. However, the dose of surfactant used in the present study was similar to that shown to be effective in a previous study [5]. In addition, the type of exogenous surfactant used in this study contained added proteins (including apoprotein B) which make the surfactant more effective at lowering surface tension [13]. The dose of surfactant was instilled directly into the back of the pharynx 
using a nasal catheter, therefore the authors are confident that the pharyngeal mucosal surface was covered.

There are few reports estimating the duration of the effects of surfactant. In the present study the RDI was measured for $1 \mathrm{~h}$ following instillation of surfactant. This $1 \mathrm{~h}$ analysis period was considerably shorter than the $3.5 \mathrm{~h}$ reported previously [6]. However, in both studies, a short half-life for the presence of surfactant could have contributed to the relatively modest effect observed.

\section{Mechanisms of action}

There are a number of possibilities that could explain the salutary effects of surfactant on the pharyngeal airway in sleeping humans. The authors considered whether the effect may be analogous to the reduction of surface tension in a sphere (Laplace Law), but they concluded that it is unlikely to be of major importance in the pharyngeal airway given its size and irregular shape. The reduction in the collapsibility of the airway is also unlikely to have been due to making the mucosal lining more moist, as there was no response after application of saline. Therefore, the authors believe that the application of surfactant delays or eliminates the phenomenon of "liquid bridging", i.e. the occurrence of a thin liquid layer that forms between two mucous folds (fig. 3).

Studies have shown that a thin liquid layer on the inner wall of a small tube can undergo fluid dynamic instability, forming a liquid bridge and eventually total occlusion of the tube [14]. In a computational model, Отіs et al. [15] showed that surfactant delays or eliminates the bridging. LIU et al. [16] also studied airflow characteristics through a narrow tube (a mouse oesophagus) when lined with saline versus surfactant. These researchers showed that when pressure was applied on one side of the tube, the air was forced through the saline bridge only for a moment because the liquid column reformed. This occurred repeatedly causing the flow to be intermittent. However, with

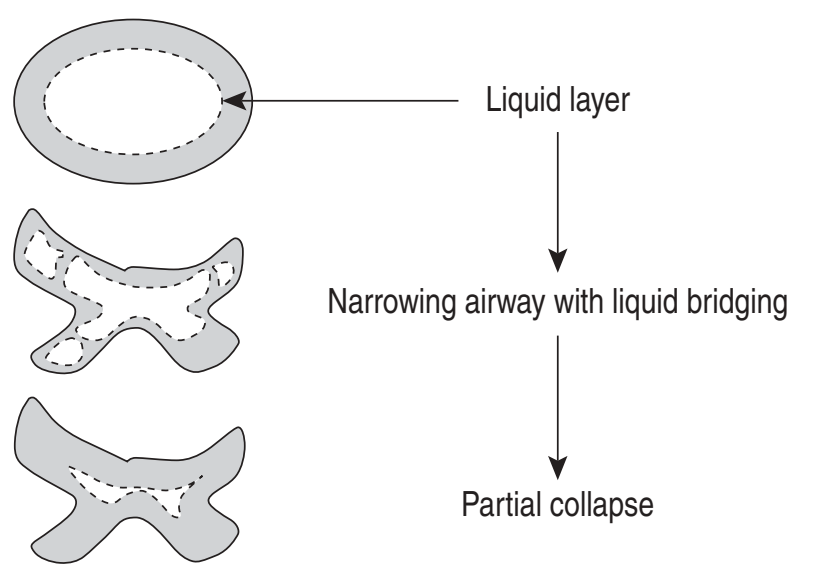

Fig. 3.-Schematic diagram of the pharyngeal lumen. Airway narrowing is associated with the formation of liquid bridging, leading to further airway occlusion. surfactant, a new liquid column did not form, and the flow remained steady. The current authors have previously observed, using flexible nasopharyngoscopy, that in some people a reduction in pharyngeal patency can occur as a result of the formation of mucosal folds; these can sometimes coalesce producing complete occlusion (for an example see fig. 2 in [17]). Here the size of the tubes formed by the mucosal folds appears to be critical. The present authors believe that as the mucosal surfaces come closer together, they start to behave as several small tubes with the formation of fluid bridging (fig. 3). In the present study as pharyngeal narrowing occurred, the surfactant decreased upper airway resistance by preventing the bridging. To further investigate this suggestion it would be important to determine the size of the tubes that could result in liquid bridging, and hence the amount of folding in the pharyngeal mucosa that predisposes to airway occlusion.

\section{Implications for sleep apnoea syndrome}

The findings of this study suggest that mucosal surface factors can contribute to pharyngeal collapse in patients with sleep disordered breathing. Interventions to reduce mucosal surface tension could be used as a therapy for patients with sleep apnoea. However, it is unlikely that such interventions will serve as a primary treatment of sleep apnoea given the modest improvement in RDI and the lack of changes in sleep patterns seen in the present study. Before considering the widespread clinical application of surface-active agents it is worth considering several points. First, the modest reduction in RDI observed would need to be confirmed in a larger, double blind, placebocontrolled trial. Second, a more clinically applicable mode of administration needs to be developed, perhaps in the form of an inhaler. Third, the preparation used was short acting, and it is likely that a longer acting preparation will be necessary to maintain the beneficial effect.

In conclusion, it has been shown that surfactant can be used to reduce pharyngeal resistance and stabilise the airway during sleep. The authors suggest that interventions to reduce mucosal surface tension may be an adjunctive therapy in some patients with mild sleep-disordered breathing.

\section{References}

1. Wilson SL, Thach BT, Brouillette RT, Abu-Osba YK. Upper airway patency in the human infant: influence of airway pressure and posture. J Appl Physiol 1980; 48: $500-504$

2. Roberts JL, Reed WR, Mathew OP, Menon AA, Thach BT. Assessment of pharyngeal airway stability in normal and micronathic infants. $J$ Appl Physiol 1985; 58: 290-299.

3. Olson LG, Strohl KP. Airway secretions influence upper airway patency in the rabbit. Am Rev Resp Dis 1988; 137: 1379-1381. 
4. Wheatley JR, Kelly WT, Tully A, Engel LA. Pressure-diameter relationships of the upper airway in awake subjects. J Appl Physiol 1991; 70: 22422251.

5. Van Der Touw T, Crawford ABH, Wheatley JR. Effects of synthetic lung surfactant on pharyngeal patency in awake human subjects. $J$ Appl Physiol 1997; 82: 78-85.

6. Jokic R, Kilmaszewski A, Mink J, Fitzpatrick MF. Surface tension forces in sleep apnea: The role of a soft tissue lubricant. A randomized double-blind, placebocontrolled trial. Am J Resp Crit Care Med 1998; 157 : $1522-1525$.

7. Hoffestein V, Mateiko S, Hallko S, Taylor R. Reduction in snoring with phosphocholinamin, a long-acting tissue-lubricating agent. Am J Otolryngol 1987; 8: 236-240.

8. Widdicombe JG, Davies A. The effects of a mixture of surface-active agents (Sonarex) on upper airways resistance and snoring in anaesthetized dogs. Eur Respir J 1988; 1: 785-791.

9. Miki H, Hida W, Kikuchi Y, et al. Effects of pharyngeal lubrication on opening of obstructed upper airway. J Appl Physiol 1992; 72: 2311-2316.

10. Rechtschaffen A, Kales A. A manual of standardized terminology, techniques and scoring systems for sleep stages of human subjects. Bethesda, Maryland, U.S. Government Printing Office, 1968.

11. Task Force of the American Sleep Disorders Association. EEG arousals: scoring and examples. A preliminary report from the Sleep Disorders Atlas Task Force of the American Sleep Disorders Association. Sleep 1992; 15: 174-184.

12. Taha B, Dempsey JA, Webber SM, et al. Automated detection and classification of sleep-disordered breathing from conventional polysomnography data. Sleep 1997; 20: 991-1001.

13. Hall SB, Venkitaraman AR, Whitsett JA, Holm BA, Notter RH. Importance of hydrophobic apoproteins as constituents of clinical exogenous surfactant. $\mathrm{Am}$ Rev Respir Dis 1992; 145: 24-30.

14. Kamm RD, Schroter RC. Is closure caused by liquid film instability? Respir Physiol 1989; 75: 141-156.

15. Otis DR, Ingenito EP, Kamm RD, Johnson M. Dynamic surface tension of surfactant TA: experiments and theory. J Appl Physiol 1994; 77: 2681-2688.

16. Lui M, Wang L, Li E, Enhorning G. Pulmonary surfactant will secure free airflow through a narrow tube. J Appl Physiol 1991; 71: 742-748.

17. Morrell MJ, Badr MS. Effects of NREM sleep on dynamic within-breath changes in upper airway patency in humans. J Appl Physiol 1998; 84: 190-199. 